\title{
Numerical study of the aerodynamic characteristics around typical terrain with different temperature
}

\author{
Xiaowei Huai ${ }^{1,2}, \mathrm{Li} \mathrm{Li}^{1,2}$, Jun Guo ${ }^{1,2}$, Shifeng $\mathrm{Wu}^{3 *}$ and Zhou Jian ${ }^{1,2}$ \\ ${ }^{1}$ State Kay Laboratory of Disaster Prevention and Reduction for Power Grid Transmission and Distribution Equipment, Changsha, China \\ ${ }^{2}$ Disaster Prevention and Reduction Center, State Grid Hunan Electric Power Company Limited, Changsha, China \\ ${ }^{3}$ College of Mathematics and Systems Science, Guangdong Polytechnic Normal University, Guangzhou, China
}

\begin{abstract}
The distributions of near-surface meteorological elements, such as wind, are greatly affected by the terrain underneath, which makes the power structure of micro geomorphic area more vulnerable to the influence of local climate. Single hills with length are one of typical terrains in microrelief. In this paper, the circulation caused by buoyant flows and temperate within typical single hilly terrain with length is studied. The Detached Eddy Simulation (DES) is used to integrate buoyancy, turbulence and micro-terrain into a single model and it is applied to the special situation of micro-terrain climate. How the wind field is influenced by different surface temperature and the model surface roughness is carefully described. The results show that, different surface temperature has a very strong effect on the speedup ratio. Compared with the air temperature, the lower the terrain surface temperature is, the more obvious the speedup ratio effect is, and vice versa. For different roughness surface terrain, the speedup ratio has almost the same characteristics.
\end{abstract}

\section{Introduction}

It's easy to have ice grow on transmission line in microrelief region than other areas due to the micro-climate caused by terrain. A simple example of how terrain affect local weather is the so called the effect of narrow where wind will speed up when passing through a narrow tube. However, there are still many challenges in providing finer resolution wind fields when considering the complicated or finer-resolution terrain in the dynamic framework of the numerical weather prediction (NWP) model (Wyngaard [1]). In recent years, a growing interest has been seen in applying Computational Fluid Dynamics $(\mathrm{CFD}$,$) to simulate complex micrometeorological pro-$ cesses in a microrelief area. According to different methods for solving the N-S equation, there are two generally accepted CFD simulation methods, namely, the Reynolds average Navier-Stokes (N-S) equation (RANS) method (Makridis[2]; Yan[3]) and the large eddy simulation (LES) method (Cao[4], Liu et al., [5], Pingzhi Fang[6]).

The study of mountain terrain wind field has been started since 1980s. Taylor[7] collated it and compared it with the theoretical calculation. It is considered that the theoretical calculation result of the acceleration ratio is far from the actual measurement result near the ground surface. Therefore, the wind speed distribution of mountain wind field still needs further study. In terms of numerical simulation, Bitsuamlak[8] collated and compared the numerical simulation and wind tunnel test of two- dimensional steep slope and gentle slope, and thought that results could better match the test results in the windward slope. However, there are two shortcomings in the above research: firstly, most of them are based on the standard model provided by Fluent, without considering the influence of factors such as buoyancy. Secondly, the influence of temperature on airflow is seldom considered in the existing literature. In the transmission line of mountain area, because the temperature difference between mountain and air changes greatly, when there is sudden wind, it is easy to happen flashover accident and galloping accident. We selected the CFD tool OpenFOAM (Weller H [9]) as development platform, incorporating the effects of buoyancy and temperature.

In this paper, aerodynamic characteristics around terrain of different roughness and with different temperature were studied numerically. The structure of this first part of the work, we briefly describe the numerical treatment used to deal with the physical problem. The governing equations and the initial and boundary conditions is considered in Section 2. One typical hilly terrain with several different temperature conditions and rough surfaces are discussed in Section 3. The results of the aero-dynamic characteristics around the various temperature models are discussed, and some conclusions are summarized in Section 4.

\section{Physical problem and model descrip- tion}

*corresponding author's e-mail: shifengwu@1sec.cc.ac.cn 
Generally speaking, the types of atmospheric circulation we study include two main processes: acceleration or return area caused by terrain or structural obstacles. The other is the buoyancy effect caused by the heat flow from or toward the surface, which can produce important vertical airflow acceleration. These convective effects determine the stability of the atmosphere and must be considered. Obviously, there is no record that OpenFOAM is used to study the circulation caused by buoyancy flow and temperature in a typical Valley, such as a single mountain model with length.

\subsection{Governing equations and implementation in OpenFOAM}

In our case, we use the quasi-compressible approximation, in which the density is taken as an explicit variable. Detailed view of algorithms for coupling pressure and speed in OpenFOAM is considered by Oliveira [10]. Considering the effect of compressibility, we have to use the filtered version of the general continuity equation (for simplicity, we do not include the bar graph above the large-scale variable):

$$
\frac{\partial \rho}{\partial t}+\nabla \cdot(\rho \vec{U})=0
$$

The momentum equation:

$$
\begin{gathered}
\frac{\partial}{\partial t}\left(\rho u_{i}\right)+\frac{\partial}{\partial x_{j}}\left(\rho u_{i} u_{j}\right)-\nabla \cdot\left(\boldsymbol{T}+\boldsymbol{T}_{\text {sgs }}\right)=-\nabla p+\rho \vec{g}- \\
\nabla p^{*}+f_{c}
\end{gathered}
$$

Where $\boldsymbol{T}$ represents the stress tensor associated with molecular viscosity

$$
\boldsymbol{T}=\mu\left[\left(\frac{\partial u_{i}}{\partial x_{j}}+\frac{\partial u_{j}}{\partial x_{i}}\right)-\frac{2}{3}\left(\frac{\partial u_{k}}{\partial x_{i}}\right) \delta_{i j}\right]
$$

while $_{\text {sgs }}$ is the Subgrid-Scale stress tensor. The OpenFOAM can offer different alternatives. The term $\nabla p^{*}$ in (2) corresponds to an external force used to maintain the flow. Although we include Coriolis $\left(\vec{f}_{c}\right)$ in $(2)$, this force is very weak in our calculation example, we do not consider it. The great advantage of OpenFOAM is that each application can be compiled independently. The thermal analysis is incorporated through an enthalpy equation,

$$
\begin{gathered}
\frac{\partial \rho h}{\partial t}+\nabla \cdot(\rho \vec{U} h)+\frac{\partial \rho K}{\partial t}+\nabla \cdot(\rho \vec{U} K)-\nabla \cdot\left(\alpha_{t} \nabla \mathrm{h}\right)- \\
\operatorname{rad}=\frac{D p}{D t}
\end{gathered}
$$

Where $h$ is specific enthalpy, $\mathrm{k}=|\overrightarrow{\mathrm{U}}| / 2$ is mechanical energy. In (4), the effective SGS thermal diffusivity, $\alpha_{t}$ is the sum of the molecular thermal diffusivity. The radiative transfer ( $\mathrm{rad}$ ) of Eq (4), which considers the use of the radiation models available in OpenFOAM(Vdovin $\mathrm{A}[11])$.In general we did not activate radiation in the our cases.

We used the ideal gas state equation:

$$
p=\rho R T
$$

with $\mathrm{R}=287 \mathrm{~J} /(\mathrm{Kg} \mathrm{K})$. To include passive temperature transport in the model, we use

$$
\frac{\partial \rho \mathrm{T}}{\partial t}+\nabla \cdot(\rho \vec{U} \mathrm{~T})-\nabla \cdot\left(\alpha_{t} \nabla \mathrm{T}\right)=f_{v}
$$

where $T$ is the temperature and $f_{v}$ is a source. is temperature in this paper. It is accepted that this property is part of the flow (Lim H[12]).

In summary, the solver includes the following equations: continuity, Navier-Stokes, and enthalpy conservation, temperature equation which are solved using libraries and tools provided by OpenFOAM.

\subsection{Initial and boundary conditions}

In CFD, the initial and boundary conditions are very important. In most cases, it is necessary to create specific conditions for each simulation, although OpenFOAM provides several predefined conditions. When we focus on the DES buoyancy simulation, we will describe these conditions in detail.

We use the logarithmic boundary conditions commonly used in atmospheric science: $U(z)=$ $\frac{U_{*}}{\kappa} \ln \left(\frac{z-z_{\text {Ground }}+z_{0}}{z_{0}}\right), \quad k=\frac{U_{*}^{2}}{\sqrt{C_{\mu}}}, \varepsilon=\frac{U_{*}^{3}}{\kappa\left(z+z_{0}\right)}$. Where $U_{*}$ (friction velocity), $\kappa$ (von Karmans constant), $z_{\text {Ground }}$ (displacement height) and $z_{0}$ (surface roughness length) are known or can be estimated from data. $K$ and $\varepsilon$ conservation equations are satisfied when $\sigma_{\varepsilon}=\frac{\kappa^{2}}{\left(c_{\varepsilon 2}-c_{\varepsilon 1}\right) \sqrt{C_{\mu}}}$

,Where $\sigma_{\varepsilon}, C_{\varepsilon 2}, C_{\varepsilon 1}, C_{\mu}$ are coefficients of the $k-\varepsilon$ model. For atmospheric flows, the roughness length, $z_{0}$ is used. The relation between $z_{0}$ and $k_{s}$ is $z_{0}=20 \mathrm{k}_{s}$. The $k_{s}$ wall functions do not accept the first cell center from the wall at a distance $k_{s}$. For low crops, $z_{0} \approx 2$ would give us $k_{s}=40 \mathrm{~m}$.

\section{Numerical Results and Discussion}

In this section, buoyant atmospheric flows over one hilly terrain with several different temperature conditions are discussed.

\subsection{Numerical Configuration}

This study focuses on the neutrally stratified boundary layer. The experimental data over three-dimensional hills with roughness surfaces based on the experiments carried out by W. Lou [13]. The typical hilly terrain have a cosine-squared cross-section as shown in Fig. 1, and are expressed as

$$
z_{S}=\left\{\begin{array}{c}
h \cos ^{2}\left(\pi \frac{r}{2 L}\right), r<L \\
0, r \geq L .
\end{array}\right.
$$

The hill height is $100 \mathrm{~m}$, The length of the hill $\mathrm{D}$ is $300 \mathrm{~m}$ and the base radius is $150 \mathrm{~m}$, and thus the slope is approximately $\mathrm{D} / L=0.6667$; 


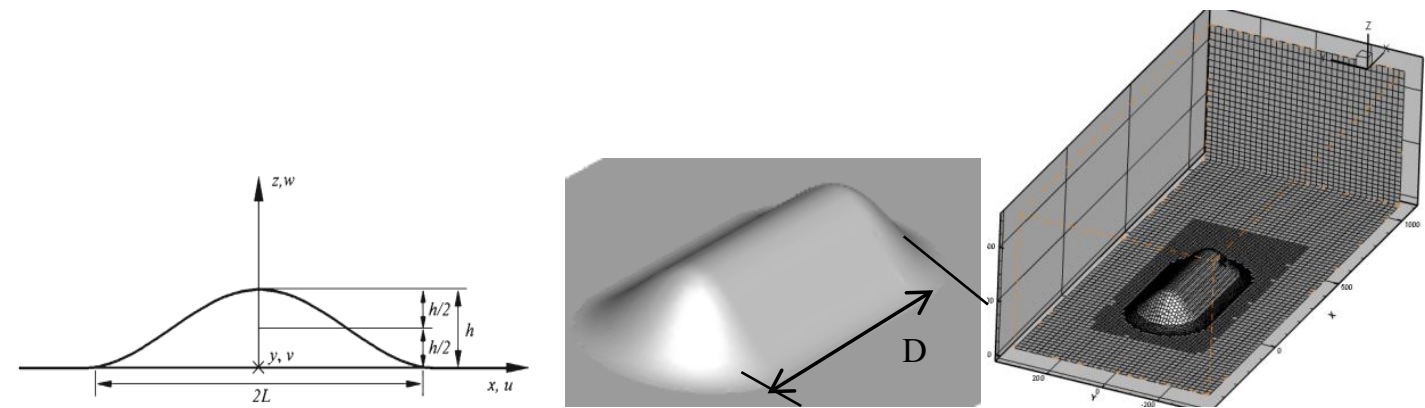

Fig. 1. Cross-section of steep ridges, hills and mesh scheme

The computational domain for the simulations with the modified DDS model is shown in Fig. 1. The size of the computational domain is $L_{x} \times \mathrm{L}_{y} \times \mathrm{L}_{z}=$ $1500 \mathrm{~m} \times 700 \mathrm{~m} \times 500 \mathrm{~m}$. Given our interest in working with complex geometries, we used the snappyHexMesh tool to solve the problem of mesh generation.

Where Laminar viscosity $\mu=1.5 e-5$, Thermal expansion coefficient beta $=3 \mathrm{e}-3$, Reference temperature TRef $=300$, Laminar Prandtl number $\operatorname{Pr}=0.7$, Turbulent Prandtl number Prt $=0.85$. Table 1 summarizes the temperature parameters used in the numerical simulations for Uref $=10.0 ;$ Zref $=10 ; z_{0}=0.1$ or $2 m ; z_{\text {Ground }}=0$. A set of experiments are simulated for different surface temperature (see Table 1).For case NoT in Table.1, which is the traditional algorithms, the influence of temperature phase and buoyant atmospheric are not considered.

\subsection{Numerical results}

The horizontal wind speed ratios $R_{i x}$ at points over the slope surface were allnormalized by the gradient velocity $U(z)$ with the expressions as follows:

$$
R_{i x}=\frac{U_{i x}-U\left(z_{i}\right)}{U\left(z_{i}\right)}
$$

where $z_{i}$ is the height above the uphill model surface at point $\mathrm{i} ; \mathrm{U}\left(\mathrm{z}_{\mathrm{i}}\right)$ is the undisturbed mean wind speed or the inflow boundary conditions at $z_{i} . U_{i x}$ is the horizontal wind speed at point $i$;

Table 1. The temperature parameters used in the numerical simulations.

\begin{tabular}{|c|c|c|c|c|c|}
\hline Case & InternalField & Outlet/inlet & Terrain & Ground & TopAndSides \\
\hline NoT & No T & No T & No T & No T & No T \\
\hline T0 & uniform 283.15; & zeroGradient; & fixedValue;273.15K & fixedVlue;273.15K & symmetry \\
\hline T10 & uniform 283.15; & zeroGradient; & fixedValue;283.15K & fixedValue;283.15K & symmetry \\
\hline T20 & uniform 283.15; & zeroGradient; & fixedValue;293.15K & fixedValue;293.15K & symmetry \\
\hline T30 & uniform 283.15; & zeroGradient; & fixedValue;303.15K & fixedValue;303.15K & symmetry \\
\hline
\end{tabular}

From Fig.2, it can be seen that the place with the largest speed ratio is between the vertical section of point $(150,0,0)$ and point $(300,0,0)$ at different temperatures. When the temperature of the mountain is higher than the air temperature (Case T20,T30), the length of the accel- eration area at the top of the mountain is obviously shortened, while when the temperature of the mountain is lower than the air temperature, the opposite is true. In point $(150,0,10)$, for case $\mathrm{T} 30$, the wind speed ratio is negative, while in case $\mathrm{T} 0$, the speed ratios is almost 1 .

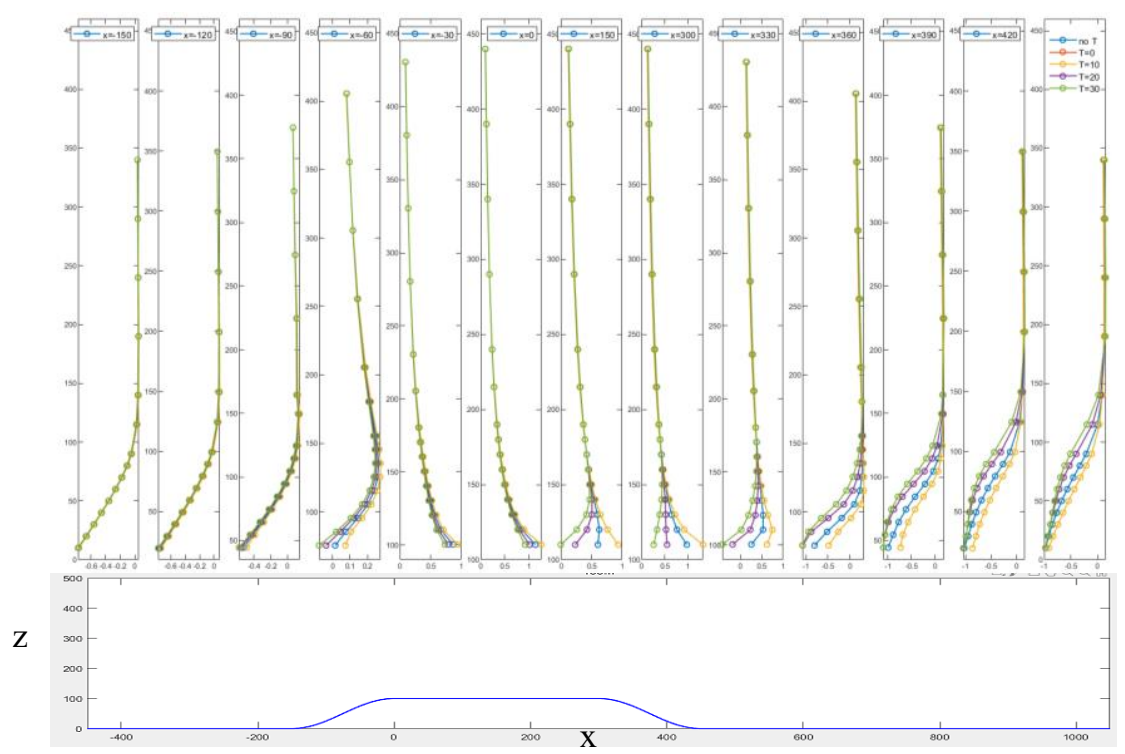

Fig. 2. Vertical profiles of wind speed ratios from the $Y=0$ section of single hill with different temperature 
(a)

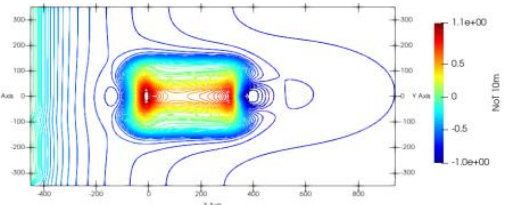

(c)

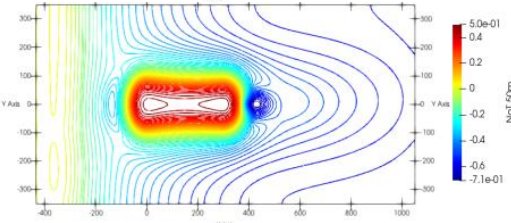

(b)

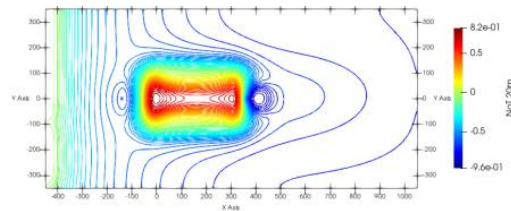

(d)

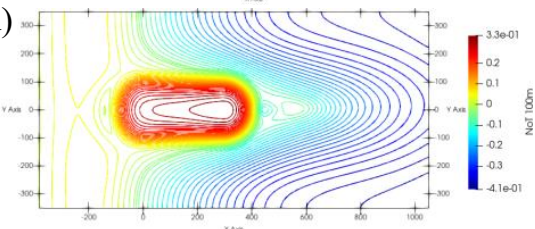

Fig3. Distribution of speed ratios $R_{i x}$ at the different height(a)10m, (b) 20m, (c)50m, (d) 100m, for Case NoT.

(a)

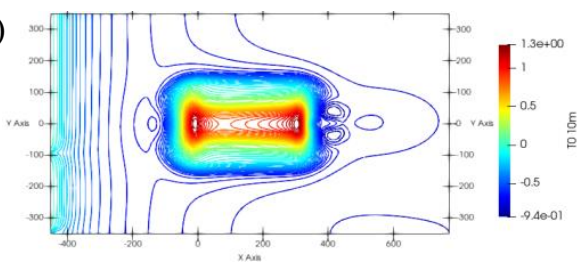

(c)

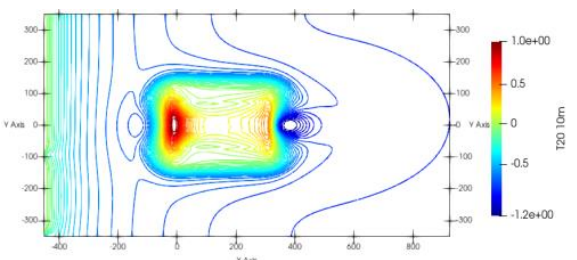

(b)

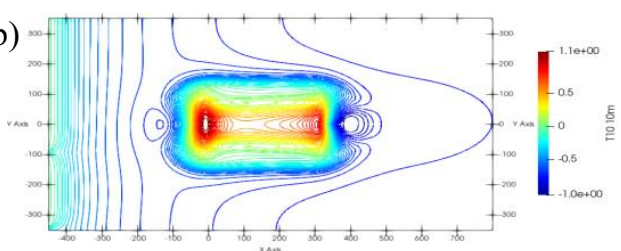

(d)

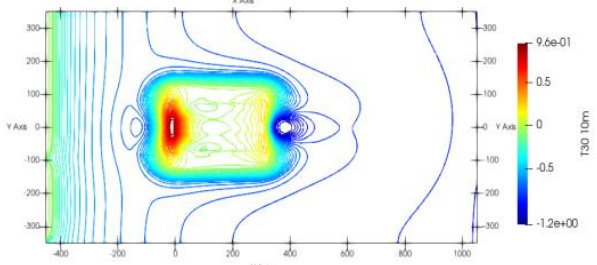

Fig.4. Distribution of speed ratios $R_{i \mathrm{x}}$ at height $10 \mathrm{~m}$ for different surface temperature (a)T0, (b) T10, (c)T20, (d)T30.

(a)

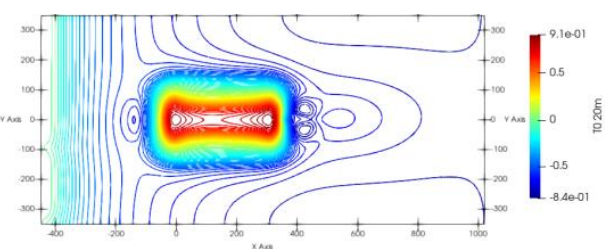

(c)

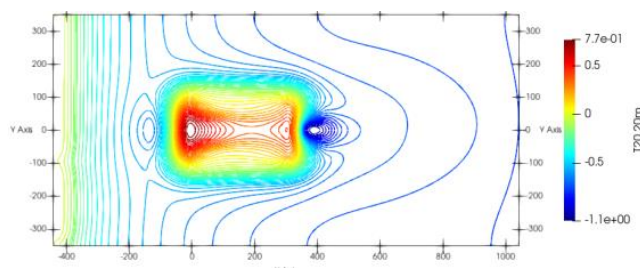

(b)

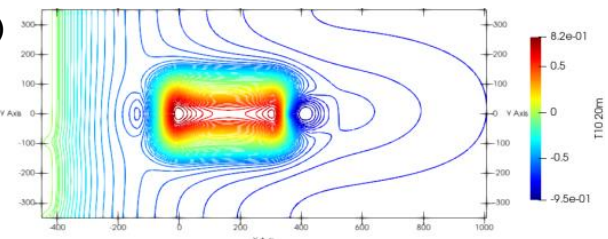

(d)

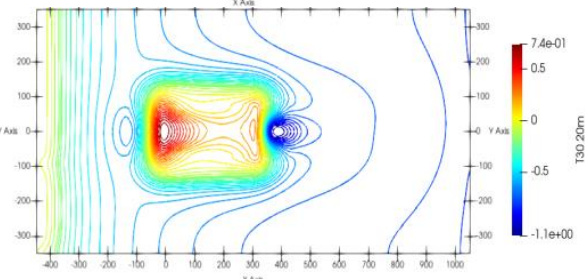

Fig. 5. Distribution of speed ratios $R_{i \mathrm{x}}$ at height $20 \mathrm{~m}$ for different surface temperature (a)T0, (b) T10, (c)T20, (d)T30.

(a)

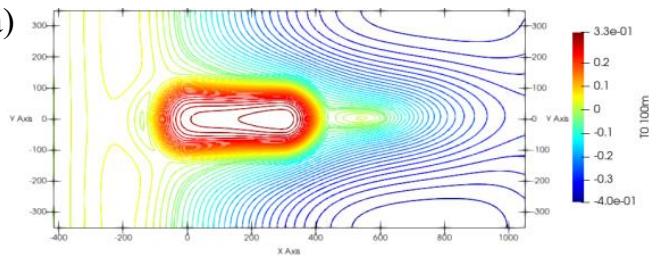

(c)

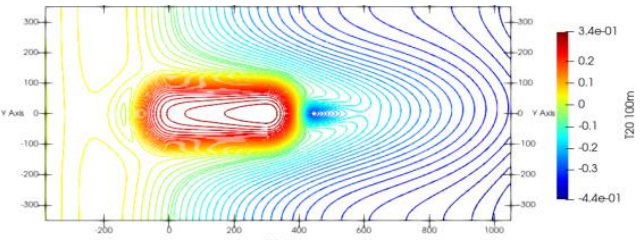

(b)

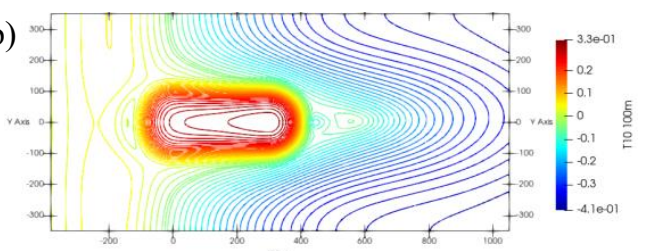

(d)

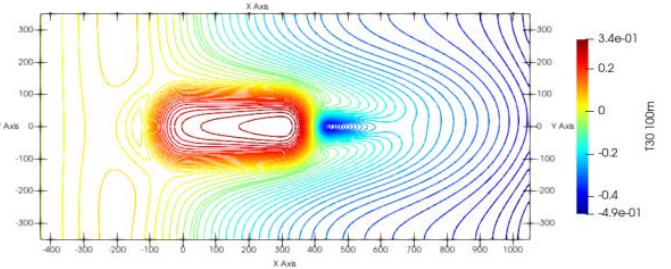

Fig.6. Distribution of speed ratios $R_{i \mathrm{x}}$ at height $100 \mathrm{~m}$ for different surface temperature (a)T0, (b) T10, (c)T20, (d)T30.

From Fig.2 to Fig. 6, it can be seen that algorithm with temperature phase and buoyant atmospheric (case T10: the algorithm uses the same temperature, so the air does not have convection heat transfer) and the tradition- al algorithm without temperature(case NoT), the conclusion is the same. It proves that our DES is correct.

It can be seen from the Fig.3-6 that when the height from the surface is more than 100 meters, there is little 
change in the speed ratios for a given number of situations. When it is less than 100 meters, different temperature has a very strong effect on the speed ratios. For different case, the place with the largest speed ratios almost appears at the top $(\mathrm{x}=0, \mathrm{y}=0, \mathrm{z}=100)$ of the windward side. For the case T0, the speed ratio of the coordinate at the top of the mountain is the largest of all. The speed ratios can be 1.3 (see Fig 2 and 3). We can also can see that: (1) In the height direction, the farther away from the mountain, the closer the speed ratios is to 0 ; (2) There is a small deceleration area at the lower height of the front of the mountain;(3) There is a large wake area at the back of the mountain;(4) The length of the acceleration region is related to the temperature at different temperatures. Generally speaking, the higher the mountain temperature is, the smaller the length of the wind acceleration area is.

From Fig.3-6, it show that the wind has obvious acceleration effect on the mountain slope, but the higher away from the mountain is, the weaker the acceleration effect is. The closer the mountain slope is, the stronger the acceleration effect is. For different temperatures, the lower the relative air temperature of mountains, the stronger the acceleration effect of wind, and the stronger the impact on the wind speed of the whole mountain. It can be seen that the case T0, the wind has a strong acceleration effect in the whole area of the mountain, while the case T30, it only has a strong acceleration effect at the top of the windward side.

\section{Discussion and conclusions}

The results of the present study can provide basic aerodynamic information to calculate the wind fields with finer resolution for a dynamical downscaling model and a parametric wind field model, some conclusions are as follows:

(1) Wind speed ratios above the terrain surface are affected by the surface temperature. When the hilly terrain temperature is lower than the air temperature, the acceleration effect of wind field on the hilly terrain surface is the most obvious. And the speedup ratio is the largest. When the hilly terrain temperature is higher than the air temperature, the acceleration effect will be weakened for a single hilly terrain with a length. The lower the temperature is, the longer the acceleration area will be.

(2) At the peak level, the biggest wind speed ratio above the hilly terrain surface reaches its maximum at the top of the windward side. The closer to the hilly terrain surface, the more obvious the wind acceleration effect is; the higher from the hilly terrain surface, the weaker the wind acceleration effect is. The wind around the hilly terrain top has obvious acceleration effect.

This study only shows the dynamic characteristics of wind speed in typical mountainous terrain under different temperature conditions, but there is still a need for further research on how to effectively consider wind speed in power engineering design.

\section{References}

1. Wyngaard J C . Toward numerical modeling in the "terra incognita". J Atmos Sci, 61(14):2004. 18161826.

2. Makridis A, Chick J (2013). Validation of a CFD model of wind turbine wakes with terrain effects. J Wind Eng Ind Aerodyn, 123(4): 2013. 12-29.

3. Yan B W, Li Q S, He Y C, Chan P W (2016). RANS simulation of neutral atmospheric boundary layer flows over complex terrain by proper imposition of boundary conditions and modification on the $\mathrm{k}-\varepsilon$ model. Environ Fluid Mech, 16(1):2016. 1-23.

4. Cao S, Wang T, Ge Y, Tamura Y (2012). Numerical study on turbulent boundary layers over twodimensional hills-effects of surface roughness and slope. J Wind Eng Ind Aerodyn, 104-106:2012,342349.

5. Liu Z, Ishihara $T$, Tanaka $T$, He $X$. LES study of turbulent flow fields over a smooth 3-D hill and a smooth 2-D ridge. J Wind Eng Ind Aerodyn, 153: 2016,1-12

6. Pingzhi FANG , Deqian ZHENG, Liang LI, Wenyong MA , Shengming TANG Numerical and experimental study of the aerodynamic characteristics around two-dimensional terrain with different slope angles。Front. Earth Sci. 2019,1-15

7. P.A. Taylor, R.J. Lee. Simple guidelines for estimating wind speed variations due to small scale topographic features. Climatolog. Bull. 1984(18), 332.

8. Bitsuamlak, GT Stathopoulos, T Bedard.Numerical evaluation of wind flow over complexterrain:review[J].Journalof Aerospace Engineering,2004,17(4):135-145.

9. Weller H, Tabor G, Jasak H, Fureby C. A tensorial approach to computational continuum mechanics using object-oriented techniques. Comput Phys 1998;12(6):620-31.

10. Oliveira P, Issa R. An improved PISO algorithm for the computation of buoyancy-driven flows. Numer Heat Transfer 2001; Part B, 40:473-793.

11. Vdovin A. Radiation heat transfer in Openfoam. Final Assignment for the Course CFD with OpenSource Software; 2009.

12. Lim H, Yu T, Glimm J, Li X, Sharp DH. Subgrid models for mass and thermal diffusion in turbulent mixing. Report, Los Alamos National Laboratory; 2009.

13. W. Lou, H. Liang, Z. Li...Vertical wind velocity distribution in typical hilly terrain. Kongqi Donglixue Xuebao/Acta Aerodynamica Sinica.2018.36(5):2018.791-797 